\title{
Hadron Spectroscopy
}

\section{Robert G. Edwards*}

Thomas Jefferson National Accelerator Facility

E-mail: edwards@jlab.org

There has been substantial progress in determining the excited state spectrum of hadrons. Recent activity has focused on extracting the resonance behavior of states, revealing how the spectrum is manifested from the theory Quantum Chromodynamics. Some of this progress is reviewed, and future opportunities are discussed.

37th International Symposium on Lattice Field Theory - Lattice2019

16-22 June 2019

Wuhan, China

${ }^{*}$ Speaker. 


\section{Motivation}

There is a resurgence of interest in hadron spectroscopy. The planned construction of GSI/Panda will have a major emphasis on spectroscopy. BES III in Beijing, an electron-positron collider, has been taking data with a major focus on heavy quark as well as light quark spectroscopy, and had produced for an unexpected right spectrum of charm mesons. The COMPASS experiment at CERN is a hadron beam experiment, and has produced some tantalizing evidence of exotic mesons. The LHCb experiment at CERN, while not necessarily a spectroscopy focussed experiment, had nonetheless produced evidence of a rich bottom-quark spectrim, including configurations of charmed baryons in five-quark states. Jefferson Lab has recently finished the $12 \mathrm{GeV}$ upgrade. The Hall B facility has a new focus on strange quark baryon spectroscopy. The newly commissioned Hall D program has a major focus on light quark meson spectroscopy using a photon bean for the production mechanism.

Most hadrons are resonances: they live for a short period of time before they decay into other particles. Experimentally, the presence of a resonance is determined through examining the energy dependence of scattering amplitudes of hadrons, where the hadrons briefly form the resonance before decaying back into separate objects. For all but the lowest lying resonances, multiple decay modes are kinematically and dynamically allowed. Typically each possible final-state is analyzed independently, and it may be that a particular resonance couples strongly to a certain channel and weakly to others. Thus theoretical suggestions for target channels can accelerate experimental progress.
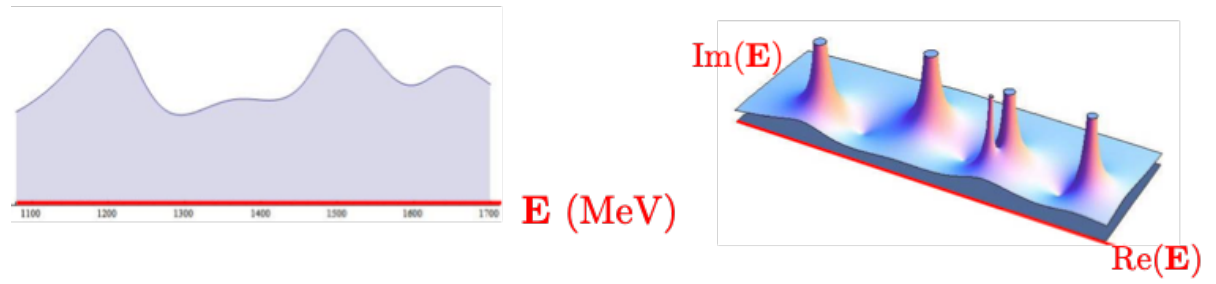

Figure 1: Left: resonances can be manifested as a strong energy dependence in real scattering amplitudes. Right: A resonance is formally defined as a pole in a partial-wave scattering amplitude.

Resonances are manifested in the behavior of scattering amplitudes. For example. there can be strong energy dependence in a real scattering amplitude as shown in Fig. 1. Resonances are formally defined as a pole in a partial-wave scattering amplitude. Different production mechanism, should have the same pole location. The pole structure, the location and residue, provides information on the decay channels. Determination of resonant content, from a first principles calculation of QCD offers both a connection between the experimentally accessible hadron observables and the fundamental quark-gluon theory, and a useful guide for experimentalists deciding where to focus their attention.

The only non-perturbative, systematically improvable method available to make such calculations is lattice QCD. To be clear, there are limitations on such calculations today. The calculations must be made with some lattice box size as well as some finite, non-zero, lattice spacing resulting in some discretization effects. Fortunately these can be systematically removed. A more important 
challenge for the present calculations attempting to determine resonance properties is the presence of a large number of thresholds for decays of high energy states. The restriction to finite spatial volume turns out to be a tool that allows us indirect access to hadron scattering amplitudes.

The formulation of scattering amplitudes within finite physical formalism, commonly referred to as the Lüscher formalism [1, 2, 3, 4, 5], provides a connection between the finite-volume energies we can compute in a lattice calculation, and geometric functions that depend on the physical volume, the total momentum of the system, and classification of the system by its irreducible cubic group of rotations.

There has been significant progress in the last few years towards elucidating the spectrum of hadrons. In this talk, we will describe some of the progress and point out future opportunities.

\section{Methods}

A comprehensive picture of resonances requires that we go beyond a determination of the ground state mass in each channel, and obtain a knowledge of the properties of a dense spectrum of excited states. This is a challenging problem. Key to progress has been the use of the variational method $[6,1,7]$ with an extensive basis of operators leading to a system of generalized eigenvalue equations for the correlation matrix. The generalized eigenvalues are solved, and their exponential dependence on the Euclidean time is used to to determined the energy of the finite-volume state.

A crucial ingredient for the success of the variational method is to use a large basis of operators in the construction of the correlation matrix. In the interacting theory of QCD, we expect the actual eigenstates to be (volume-dependent) admixtures of what we might call "single-hadron" basis states and multi-hadron basis states. It is useful to introduce operator constructions that might produce such states. An effective approach, demonstrated over a series of papers [8, 9, 10, 11, 12], is to consider fermion bilinears built using up to some number of gauge-covariant derivatives acting on the fermion fields for "single-meson" operators, and three quark constructions for "single-baryon" operators [13, 14]. In addition, multi-hadron constructions are crucial.

It is the addition of multi-particle operator constructions into the basis that allow us to resolve the scattering states that are present in finite-volume calculations. There now have been several successful demonstrations of the efficacy of this approach in resonance determinations $[15,16,17$, $18,19,20,21,22,23]$. The $P$-wave isospin=1 $\pi \pi$ scattering in the $\rho$ channel is the prototypical test case.

After integration of the quark fields within the matrix of correlation functions, there can be a large number of graph topologies. The "distillation" technique [24, 25] provides a powerful framework for their calculation. In this approach, a low-rank representation of the quark smearing operator is used whose purpose is to enhance the low momentum quark and gluon modes that dominate low mass hadrons. The method allows a factorization of the correlation functions into products of objects describing quark propagation as well as objects representing hadron operators. Graph optimization techniques can be used to efficiently compute these graphs [26, 27].

Finally, we exploit methods relating the energy spectrum of the theory in a finite-volume to energy- dependent hadron scattering amplitudes in infinite volume, which can be analyzed for their resonant content. Many derivations of the coupled-channel extension exists [28, 29, 30, 31, 32]. 
There also derivations in specific channels or for specific parameterization of the interactions. A review of the formalisms is available in Ref. [33].

An underlying issue, though, is that the quantization conditions - a determinant involving a suitable $K$-matrix for the infinite-volume amplitude and geometric functions - provides only one constraint for a specific energy and cubic group irreducible representation of the system. For a coupled channel system, there will be in general more unknowns than there are unknowns parameterizing the amplitudes. The parameters are under constrained. One way forward is to consider parameterizations of the energy dependence of the scattering $t$-matrix, at least over some interval of energies. In the phenomenological community, this is called an "energy dependent" solution of the $t$-matrix. Many of the results presented here will use this formulation.

We note that many-body resonances, beyond two-body, can open over some desired region of investigation. To investigate, let us consider the canonical test case for resonance studies - the isospin=1 $P$-wave scattering in $\pi \pi$ in the $\rho$ channel. In [34], it was found that the $4 \pi$ threshold is below $\bar{K} K$ for a pion mass of $236 \mathrm{MeV}$. Rigorously, we should be considering this open channel, but the current multi-particle formalism has not advanced to this stage. What can we say? We do not know the precise form of the equation that describes $2 \pi-4 \pi$ scattering, but we know it must have the following form [35]:

$$
\operatorname{det}\left[\left(\begin{array}{cc}
\mathscr{M}_{2 \pi} & \\
& \mathscr{M}_{4 \pi}
\end{array}\right)^{-1}+\left(\begin{array}{ll}
\tilde{K}_{2 \pi, 2 \pi} & \tilde{K}_{2 \pi, 4 \pi} \\
\tilde{K}_{4 \pi, 2 \pi} & \tilde{K}_{4 \pi, 4 \pi}
\end{array}\right)\right]=0,
$$

where $\mathscr{M}$ are the finite-volume geometric functions and $\tilde{K}$ are entries for a suitable $K$-matrix related to the infinite-volume $t$-matrix. If $\tilde{K}_{2 \pi, 4 \pi} \sim \mathscr{O}(\varepsilon)$, then Eq. 2.1 factorizes into

$$
\operatorname{det}\left[\mathscr{M}_{2 \pi}^{-1}+\tilde{K}_{2 \pi, 2 \pi}\right] \times \operatorname{det}\left[\mathscr{M}_{4 \pi}^{-1}+\tilde{K}_{4 \pi, 4 \pi}\right]+\mathscr{O}(\varepsilon)=0 .
$$

Thus, if the $4 \pi$ determinant where to generate zeros then a simple fit guided by the $2 \pi$ determinant condition should fail. We do not see such a failure, and as shown in Fig. 2, the fits for $2 \pi$ (and $\bar{K} K$ appear reasonable, thus there is no clear evidence of contributions from $4 \pi$.

Clearly, we would like to have the formalism in place so that we can rigorously include channels of high particle multiplicity. In the meantime, we at least have a guide. If high-multiplicity channels only weakly interact in a partial wave, we should, in general, expect to see extra levels in the spectrum close to their non-interacting multi-particle energies. These extra levels will likely be observed in the overlaps of the multi-particle creation operator with the finite-volume state.

\section{Light mesons}

Conventional wisdom says the light scalar mesons form an "inverted" mass nonet. However, are the mesons actually this similar? If so, empirically, they have vastly different imaginary parts. For example, the $\sigma(500)$ and $\kappa(700)$ have an imaginary part of their poles $\approx 500 \mathrm{MeV}$, while the $a_{0}(980)$ and $f_{0}(980)$ are narrower and appear near $\bar{K} K$ threshold. What does QCD have to say?

While direct calculations in the physical limit of QCD are possible today, the complication is the finite-volume formalism. In the physical limit, there will will be a large number of thresholds open to study these systems, including $4 \pi$, where the existing formalism is not valid. A way to 


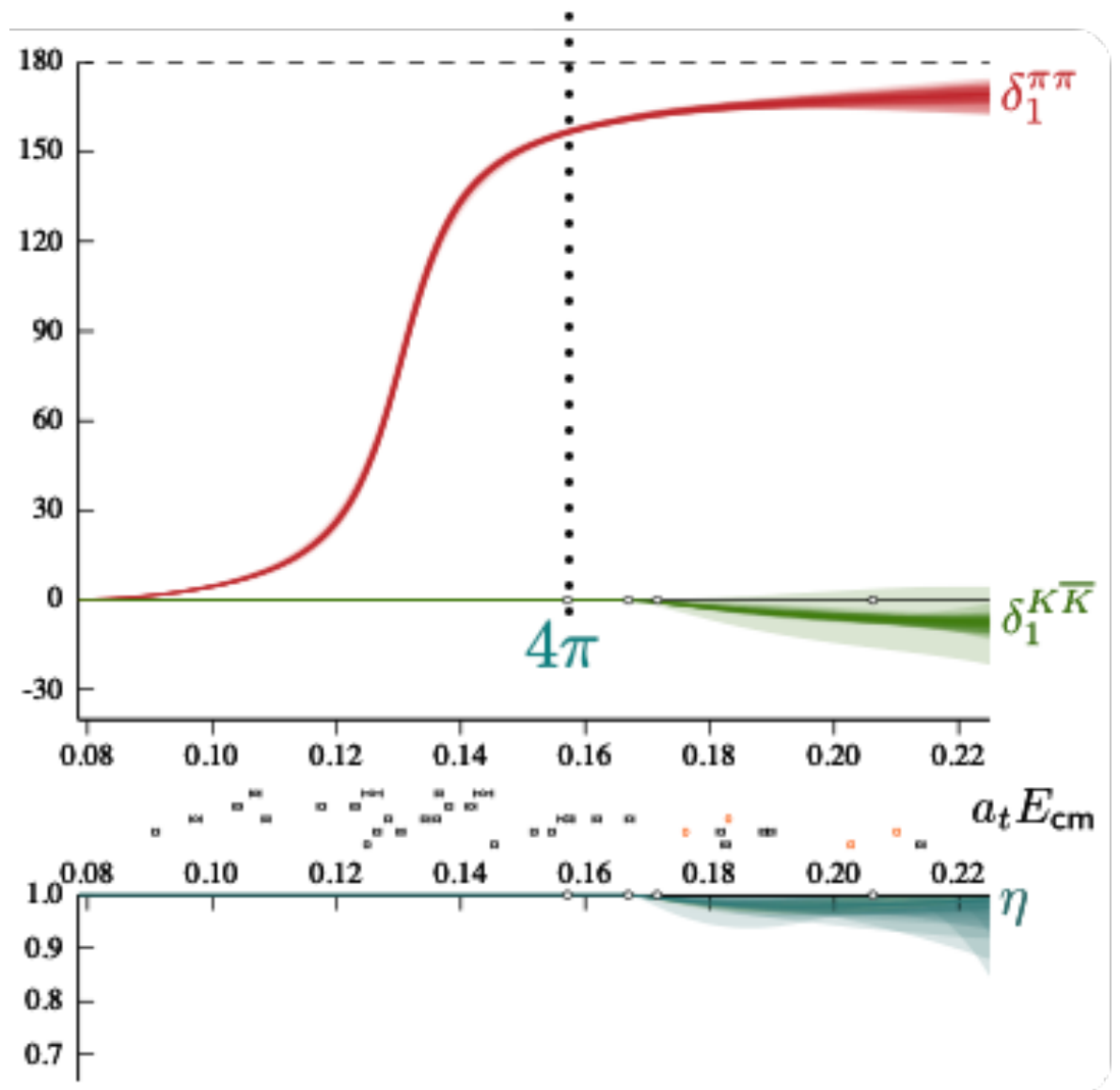

Figure 2: Results from [34]. Coupled $P$-wave $\pi \pi$ and $K \bar{K}$ isospin=1 phase shifts Ît' and inelasticity Î̉ from a single K-matrix fit. Statistical uncertainty shown by the shaded band. The central points show the energy levels constraining the amplitude ex- traction with those dominated by $\pi \pi$-like and $\bar{q} q$-like operators shown in black and those with significant $K \bar{K}$ contributions shown in orange. On axis circles show the opening of the $4 \pi, K \bar{K}, \eta \pi \pi$ and $\pi K \bar{K}$ thresholds.

make some initial progress is to dial up the quark mass such that these multi-particles thresholds are pushed higher in energy. Thus, we can expect to use the finite-volume in the regime where it is rigorously established.

Let us first consider the lightest tensors at a heavy pion mass of $m_{\pi}=391 \mathrm{MeV}$ from Refs. [36, 26, 37, 38, 39]. Shown in Fig. 3 are the lightest tensor scattering amplitudes for isospin 0, 1, and $\frac{1}{2}$. The top panel shows the isoscalar sector where there clear is enhancement in $\pi \pi$ and in $\bar{K} K$ at slightly higher energy. The $\eta \eta$ levels seem to decouple, and there is no observed effect from the presence of the $\eta \eta$ threshold. The behavior in the $\pi \pi, \bar{K} K$ sector appears to be that of two narrow resonances, with the ligher decaying primarily into $\pi \pi$ and the heavier mainly to $\bar{K} K$. The is a small amount of inelasticity. There are corresponding poles shown in the bottom left panel with the $f_{2}^{a}$ broader and at lower energy compared to the $f_{2}^{b}$. This behavior is reminiscent of the experimentally observed $f_{2}(1270)$ and $f_{2}(1525)$ with corresponding branching fractions of $84 \%$ to $\pi \pi$ and $89 \%$ to $\bar{K} K$. This is an example of flavor tagging by decays.

In the middle right panel we see a rapid change in phase for the isovector tensor suggesting a 

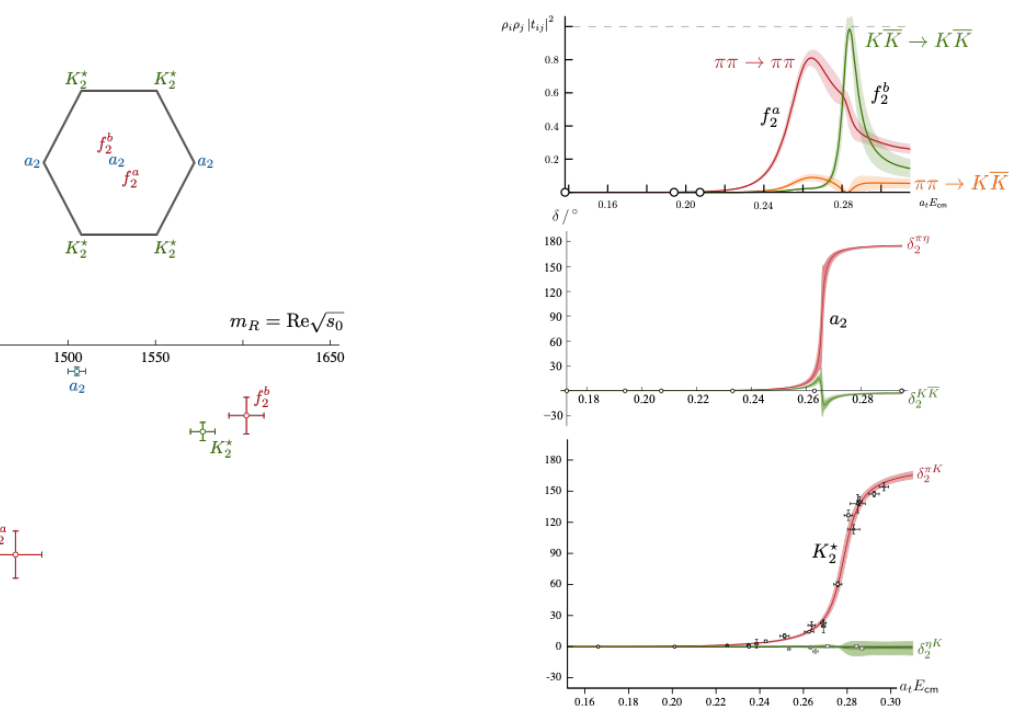

Figure 3: Results from Refs. [36, 26, 37, 38, 39]. Shown is the characterization of the lowest tensor nonet of mesons. In the right panels are scattering amplitudes and phase shifts in isospin $I=0$ (top), $I=1$ (center), and $I=\frac{1}{2}$. Pole locations are shown in the bottom left panel.
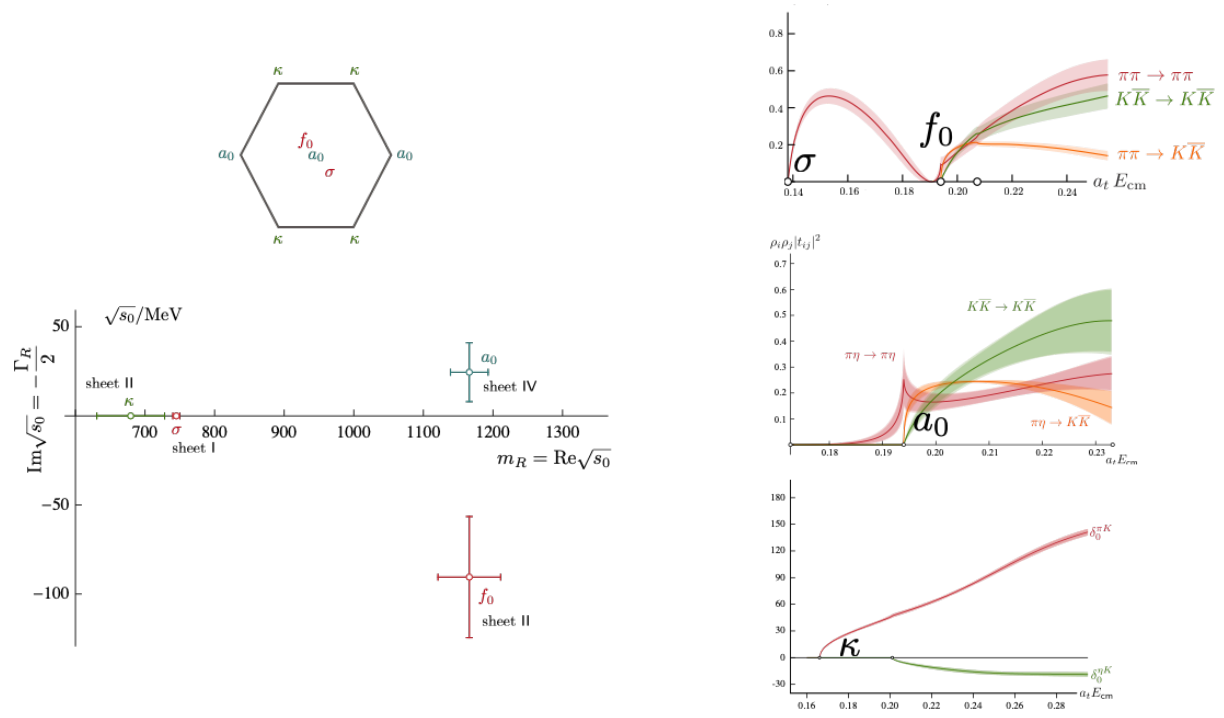

Figure 4: Results from Refs. [36, 26, 37, 38, 39]. Shown is a characterization of the lowest scalar nonet of mesons. In the right panels are scattering amplitudes and phase shifts in isospin $I=0$ (top), $I=1$ (center), and $I=\frac{1}{2}$. Pole locations are shown in the bottom left panel. 
narrow resonance, coupled to both $\eta \pi$ and $\bar{K} K$. Indeed, a pole is found close to the real axis. A narrow resonance is also observed in isospin $\frac{1}{2}$ with strong coupling of $K_{2}$ to $\pi K$. The picture thus assembled for the light tensors fits reasonably well into a grouping of nine states.

Of course, The term "octet" is an $S U(3)_{F}$ construction which is broken by our dynamical $N_{f}=2+1$ calculations. Calculations directly and slightly away from the $S U(3)_{F}$ would inform as how the mesons are grouped, and the evolution with quark mass would instruct us whether $S U(3)_{F}$ flavor is a good representation of the isoscalar scalar sector.

Switching our attention to the lightest scalars, again at the same pion mass of $m_{\pi}=391 \mathrm{MeV}$, we see a fairly different picture $[36,26,37,38,39]$. Shown in Fig. 4 are the lightest $S$-wave scalar scattering amplitudes. In the top right panel, we see a strong energy dependence in isospin $=0 \pi \pi$ with a dip appearing, and then a rapid turn on in $\pi \pi$ and $\bar{K} K$. There appears to be a bound state pole corresponding to a $\sigma$ and a pole found for a $f_{0}$ near $\bar{K} K$ as shown in the lower left panel of Fig. 4.

In isospin=1, we see in the right center panel a rapid turn on of the $\bar{K} K$ amplitude at threshold, and a rapidly varying strength in $\pi \eta$. There appears to be a single pole found on sheet IV corresponding to an $a_{0}$, meaning the pole location is closest to $\bar{K} K$. In contrast, in the lower right panel, we see a very slowly rising amplitude in $\pi K$ with a virtual bound state pole found corresponding to a $\kappa$.

Taken all together, we have a picture of the light meson resonances at a pion mass $m_{\pi} \sim$ $391 \mathrm{MeV}$. In Fig. 2, there appears to be a lowest scalar, vector and tensor grouping of states that has the counting of a nonet; albeit with fairly different pole behavior.

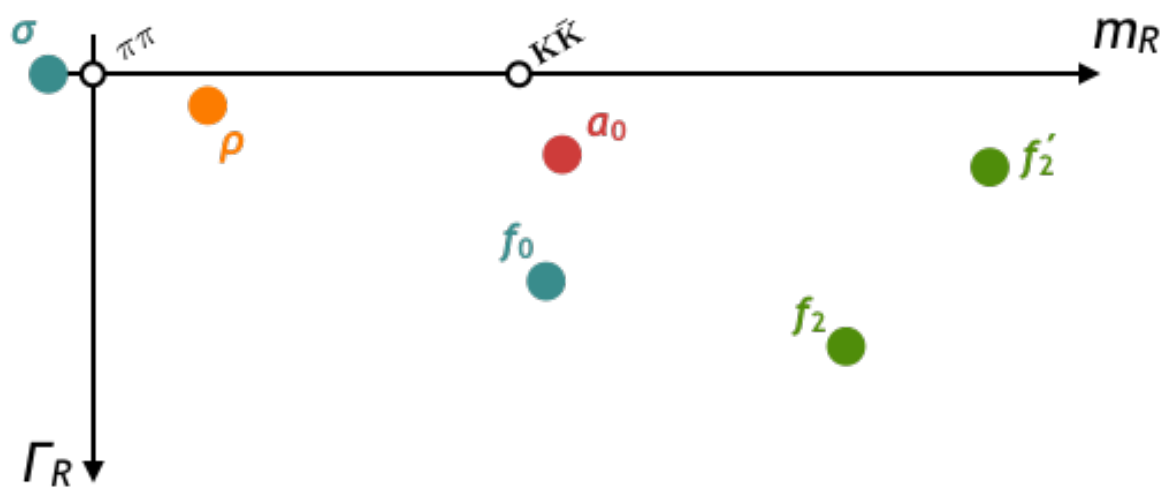

Figure 5: Collecting information from Figs. 3 and 4. Cartoon representation of the observed light meson resonances poles at $m_{\pi} \sim 391 \mathrm{MeV}$.

Next, we study the quark mass dependence of the light mesons. In the top right panel of Fig. 6, we see in isospin $=1 \pi \pi$ scattering in $P$-wave that the phase shift broadens as we move from a pion mass of $m_{\pi}=391 \mathrm{MeV}$ down to $236 \mathrm{MeV}$. In the top left panel is shown the extracted pole location at several pion masses, we see a trend toward the phenomenological value [40]. We note the coupling of the pole into $\pi \pi$ is fairly independent of the quark mass.

In the bottom with panel of Fig. 6 , we see the phase shift shown as $p \cot \delta_{0}$ for isospin $\pi \pi$ $S$-wave scattering. There is a transition to a very broad resonance as shown in the lower left panel. The coupling into $\pi \pi$ is fairly insensitive in quark mass dependence [38]. 

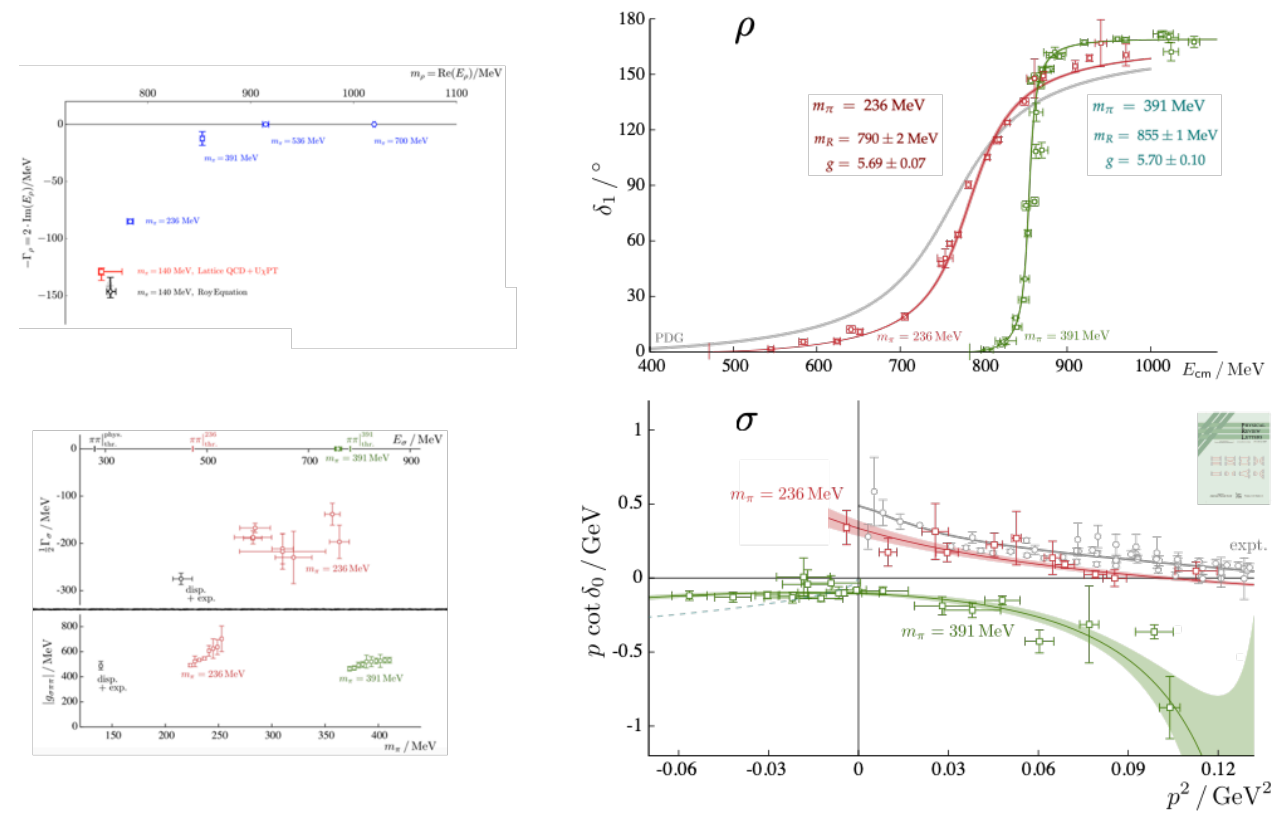

Figure 6: Results from Refs. [34, 38]. Top right: the quark mass dependence in isospin $1 P$-wave $\pi \pi$ phase shift. Top left: location of the extracted resonance pole over a range of pion masses. Bottom right: $p^{3} \cot \delta_{0}$ versus 4-momenta of the isospin=0 $\pi \pi S$-wave phase shift. Bottom left: different parameterizations of the analytic behavior of the $S$-wave amplitude. Also shown are the couplings.

In Fig. 7 we show the quark mass behavior of the $S$-wave and $P$-wave phase shifts in isospin $=\frac{1}{2}$ scattering in $K \pi$ over a wide range of pion mass ensembles. We see, in general, a broadening of the phase shift as the pion mass is reduced. The $K^{*}$ shows a very clear mass dependence. As the pion mass is decreased, the pole location moves on the complex towards the phenomenologically determined value as shown in the lower left panel. The coupling, however, is insensitive to the change of the quark mass.

The resonance behavior of the $\kappa$ is more complicated. While the phase shifts are well determined, there is not enough constraints on the analytic behavior of the amplitude to confidently extract a pole location and coupling. We expect that there is a left hand circular cut in $S$-wave scattering. This analytic behavior should be incorporated into scattering amplitudes [41].

\section{Baryons}

There has been progress in resonance determinations in baryon systems. Besides the increased computational cost in hadrons with three quark lines as opposed to meson systems, the spectrum of baryons is dense for a fixed isospin and parity channel. To adequately determine scattering amplitudes, say in $N \pi$, and hence resonance behavior, moving frames are needed to provide energy levels to constrain the amplitudes. Typically this means that one will be considering both positive 


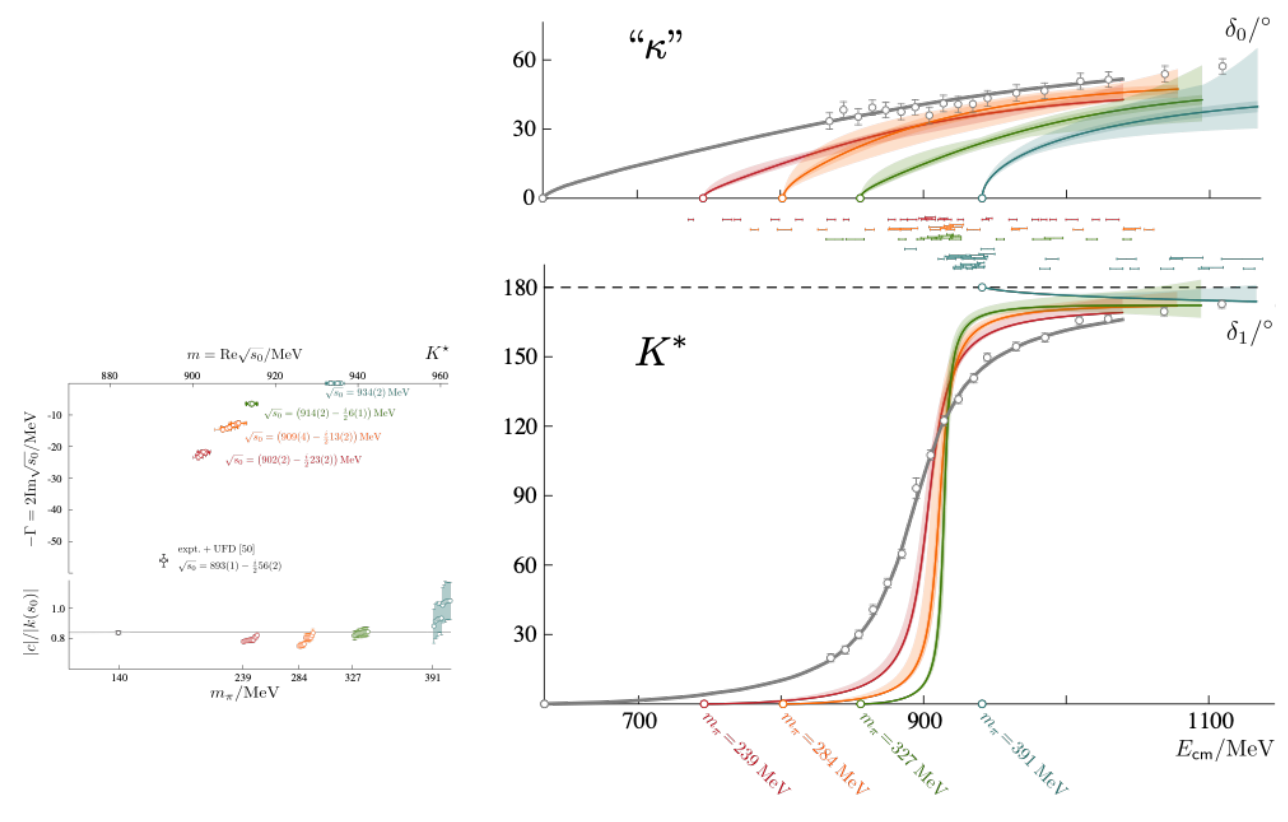

Figure 7: Results from Ref. [41]. The quark mass dependence in the isospin $=\frac{1}{2}$ (top) $S$-wave and (borrom) $P$-wave phase shifts. The left panel shows the quark mass evolution of the $K^{*}$ pole location as well as resonant coupling.

and negative parity partial waves. Depending on the isospin, say in the nucleon, one might have to contend with the nearby threshold of $N \eta$, and hence one must consider coupled channels.

A first place to disentangle these issues is to consider the low energy behavior of isospin $=\frac{3}{2}$ scattering of $N \pi$ corresponding to the $\Delta(1232)$ resonance. Utilizing the stochastic variant of distillation, it has been found [42] that at a pion mass $m_{\pi} \sim 280 \mathrm{MeV}$, that one can determine a Breit-Wigner amplitude straddling the $N \pi$ threshold and extract a coupling that is close to the phenomenological value. More investigations are warranted.

\section{Charmonium}

There has been considerable experimental activity in the charmonium sector. LHCb has been particularly active, and has reported a new state in $J^{P C}=3^{--}$. This state can fit into a conventional charmonium picture. The added difficulty for a lattice calculation to extract a state is that multiple partial waves, namely, $\ell=1$ and 3 must be determined. A first study of this system [43] has presented encouraging results.

There has been excitement generated with the announcement of charmonium states with isospin $=1$. Such states must necessarily involve light quarks along with the charm and anti-charm quarks. A "tetraquark" configuration is a possible explanation. From a lattice QCD perspective, the calculation of the finite-volume energies should include an extensive operator basis. Two-mesons with some relative momentum will have little overlap onto a single site four-quark construction. A 
single-site tetraquark operator construction can supplement the basis. Preferably, we would like to have a full scattering calculation involving multiple frames. However, a first pass can elucidate whether the inclusion of tetraquark operators affects the finite-volume spectrum, or rather, have strong overlap onto finite-volume levels not reliably determined with only meson-meson levels. A recent calculation [44] has explored the spectrum of the isospin=1, G-parity plus, and $J^{P}=1^{+}$and $0^{+}$. This sector is where the tentative $Z_{c}$ appears. It was found that the tetraquark operators do not provide additional overlaps. In addition, there does not appear to be any strong evidence of interactions generating resonant behavior.

\section{Coupling resonances to currents}

Electromagnetic probes provide a way to analyze the internal structure of resonant states. There have been studies of the time-like pion form-factor of $\gamma^{*} \rightarrow \pi \pi$ in Refs. [45, 46] as well as the $\rho$ transition form-factor in $\gamma^{*} \pi \rightarrow \pi \pi$ in Refs. [47, 48].

A key point of these studies is that the resonant pole location for the isospin $=1$ channel, the $\rho$, should be independent of the process. The couplings are dependent on the process. Indeed, within the $\pi \pi$ energy dependence of the form-factor, one observes near the resonance an enhancement of the $\pi^{+} \gamma \rightarrow \pi^{+} \pi$ cross-section.

The $P$-wave $\rho$ system is a fairly simple system. These investigations establish the feasibility of studying the radiative transition of two-body hadronic, and even nuclear systems, directly from QCD. The technology for studying transition amplitudes with any number of open two-body states has been developed $[49,50]$. We anticipate there will be more extensive investigations of other systems in the future. Some obvious targets are the many-quark systems recently reported in the charm sector.

\section{Pentaquarks}

Recently, LHCb has reported evidence of two pentaquark $\left(P_{c}\right)$ states [51, 52]. The $J^{P}$ where not determined, but it was inferred that the two resonances were either $J^{P}= \pm \frac{3}{2}, \mp \frac{5}{2}$. The complication is that these states are observed in three-body decays $\Lambda_{b}^{0} \rightarrow J / \psi p K^{-}$. These are complicated analyses. To reconstruct the events, there are more than ten Breit-Wigner resonances that are considered in the invariant mass in $K p$. New results reported in [53] suggest that there are actually three state close to thresholds as shown in the left panel of Fig. 8.

Recently, GlueX has reported [54] a study of threshold $J / \psi$ in the channel $p \gamma \rightarrow P_{c}^{+} \rightarrow J / \psi p$. If there are indeed resonances in $J / p s i p$, then the pole should be independent of the process, as emphasized earlier. The advantage to study the threshold $J / p s i$ is that the process can be reconstructed as a two-body decay. As shown in the right panel of Fig. 8, there is no clear enhancement of the cross-section. This can suggest that the photo-coupling is small. However, by employing a vector-meson dominance model of the photon, and using the decomposition of the photon current into a $\bar{c} c$ component, some constraints can be placed on the resonant production of $J / p$ sip directly. For example, the upper limit of the branching fraction of $\mathscr{B}\left(P_{c}^{+}(4400) \rightarrow J / p s i p\right)$ is found to be a few percent. While these results do not rule out a pentaquark, they do place strong constraints 

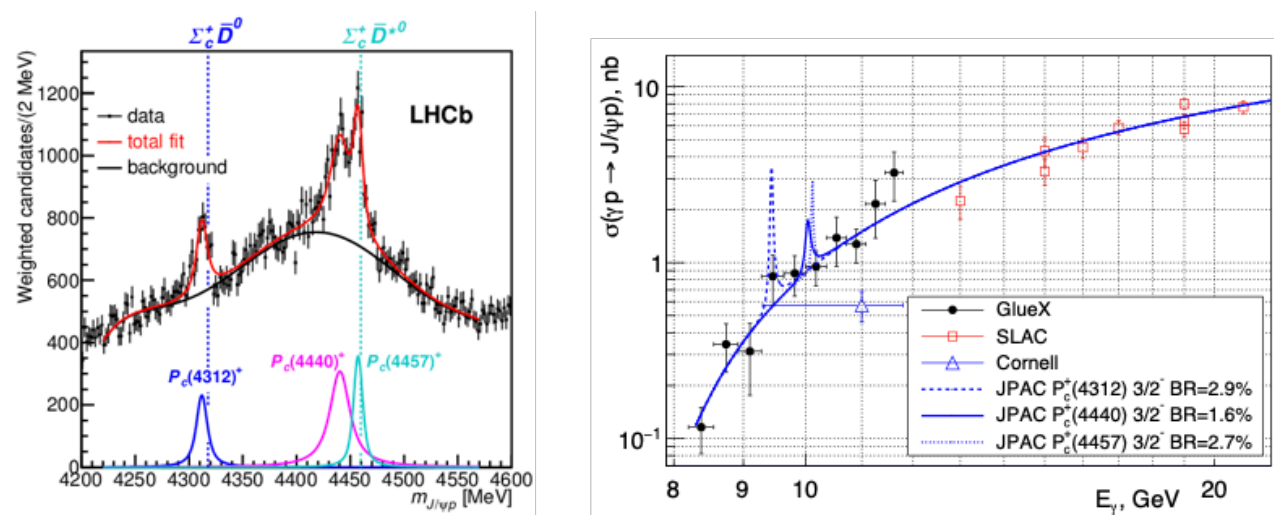

Figure 8: Left: $\mathrm{LHCb}$ [53] has reported three states close to thresholds. These are candidates for pentaquarks $P_{c}$. Right: Photo-production cross-section measurements by GlueX [54] of $p \gamma \rightarrow J / \psi p$.

on their structure and some of the models put forth to describe their behavior. Direct calculations from QCD would enlighten our understanding.

\section{8. $2+\mathscr{J} \rightarrow 2$ transition amplitudes}

As lattice has, in principle, access to the full $S$-matrix of QCD can, we can calculate quantitates that, while not directly accessible to experiment, still allow us to probe the theory and gain insight on the collective behavior of quarks and gluons. One opportunity afforded to us through the recent development of the finite-volume formalism scattering formalism of a current insertion in a twoparticle scattering system [55]. These methods open the a way to probe in the internal structure of resonances, for example, charge radii, form-factors and even parton distribution-functions [56].

Perhaps not surprisingly, there is a great deal of similarity between the formalism for $2+$ $\mathscr{J} \rightarrow 2$ scattering with that for three-particles in $3 \rightarrow 2$. A workflow of inputs and relations is shown in Fig. 9. For example, in the system $\gamma^{*} \pi \pi \rightarrow \pi \pi$, we expect the amplitude to have the functional dependence $\mathscr{A}\left(E_{\pi \pi}^{\text {in }}, E_{\pi \pi}^{\text {out }}, Q^{2}\right)$. Of course, in a finite-volume, there is a discrete spectrum of energies. To fully map the form-factor, we must calculate radiative transition form-factors from the different energy levels of the in and out states. We thus can expect to see some strong interplay between the energy of the initial/final state and the $Q^{2}$.

For a system such as the $a_{0}(980)$ which appears in strongly coupled $\eta \pi$ and $\bar{K} K$, we might hope to disentangle whether such a state is "molecular" in origin. Indeed, there are opportunities to resolve properties of possible charmonium tetraquark and pentaquark states.

\section{Status of scattering formalisms}

As noted, a great deal of progress has been made in establishing the finite-formalism of twobody coupled channel scattering. Within this framework, determinant conditions relate infinitevolume Minkowski scattering amplitude to certain geometric functions. The amplitude formalism for two-body channels is fairly well established, but what is lacking is a full understanding of how the presence of a left-hand cut can be included into scattering amplitudes while also maintaining full unitarity as well as crossing symmetries. There are further opportunities in this area. 


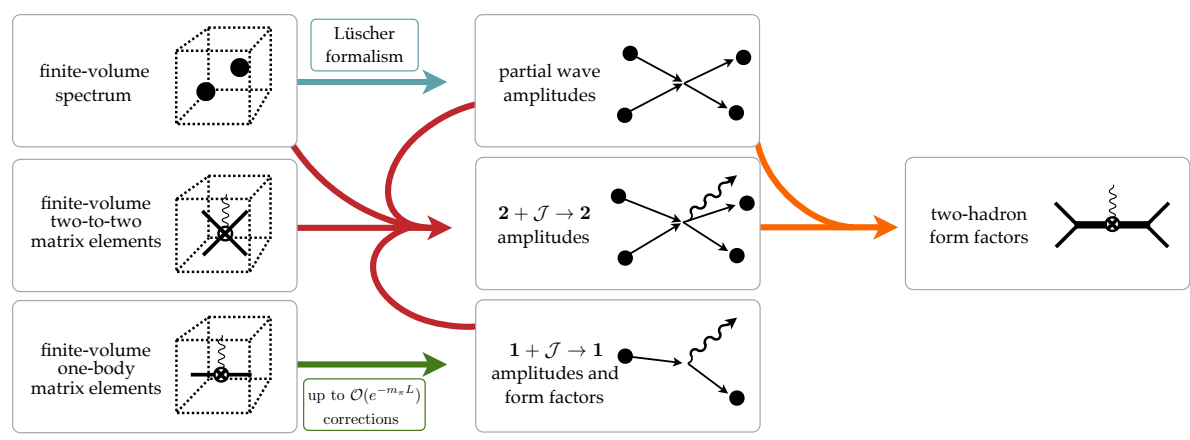

Figure 9: Graph taken from [55]. Inputs and relations for the $2+\mathscr{J} \rightarrow 2$ scattering formalism to determine a two-particle form-factor. Inputs include the single-particle form-factors and the finite-volume spectrum and determination of the two-particle scattering amplitudes.

There has also recently been considerable progress in establishing a full three-body scattering formalism. There exists a three-body finite-volume formalism in place for spinless particles supporting coupled two-body and three-body amplitudes [57] with a determinant condition that resembles two-body. Namely, the determinant is amongst the inverse of a K-matrix plus a matrix of geometric functions. The K-matrix can be related to the full scattering amplitude through a set of integral equations. It has been shown that infinite-volume three-particle scattering amplitudes arising from a finite-volume satisfies unitarity [58].

What is not well understood is three-body amplitude formalism. There has been comparison and cross-checks of existing forms [59]. Other approaches include building in to some extent additional symmetries or constraints on the scattering system that then can lead to a three-body relation to the finite-volume energies that can be extracted from a lattice calculation [60, 61, 62]. What is needed are calculations in three-body systems to drive this development. In this regards, there also has been considerable progress in making tractable - and computationally efficient - the calculation of Euclidean correlation functions [44, 63, 27].

\section{The role of glue}

The early, but extensive, calculations of the spectrum of hadrons has revealed there is a rich spectrum of states, including the possible existence of exotic and hybrid states $[64,65,66,13,67$, $68,69]$. A basic pattern seen in the spectrum of light, strange and charm quark mesons and baryons is that it is consistent with a picture of constituent quarks and antiquarks, but with the important addition of gluonic excitations. The counting and multiplicity of the spectrum is consistent with the addition of a constituent gluon in a chromo-magnetic color octet in $J^{P C}=1^{+-}$configuration. The states found in the spectrum that are assigned as hybrid in content have strong overlap with operators featuring the commutator two covariant derivatives placed. Shown in Fig. 10 is the spectrum of the hybrid states where a quark-mass scale is subtracted. For a light quark meson, this is the $\rho$ mass, for light quark baryons, it is the nucleon mass.

There is a common energy scale of gluonic excitations at about $1.3 \mathrm{GeV}$ over a wide range of pion masses as well as channels, including isovector and isoscalar mesons, light quark masses, and also charmonium mesons. As mentioned, these states have a strong overlap of gluonic operator 


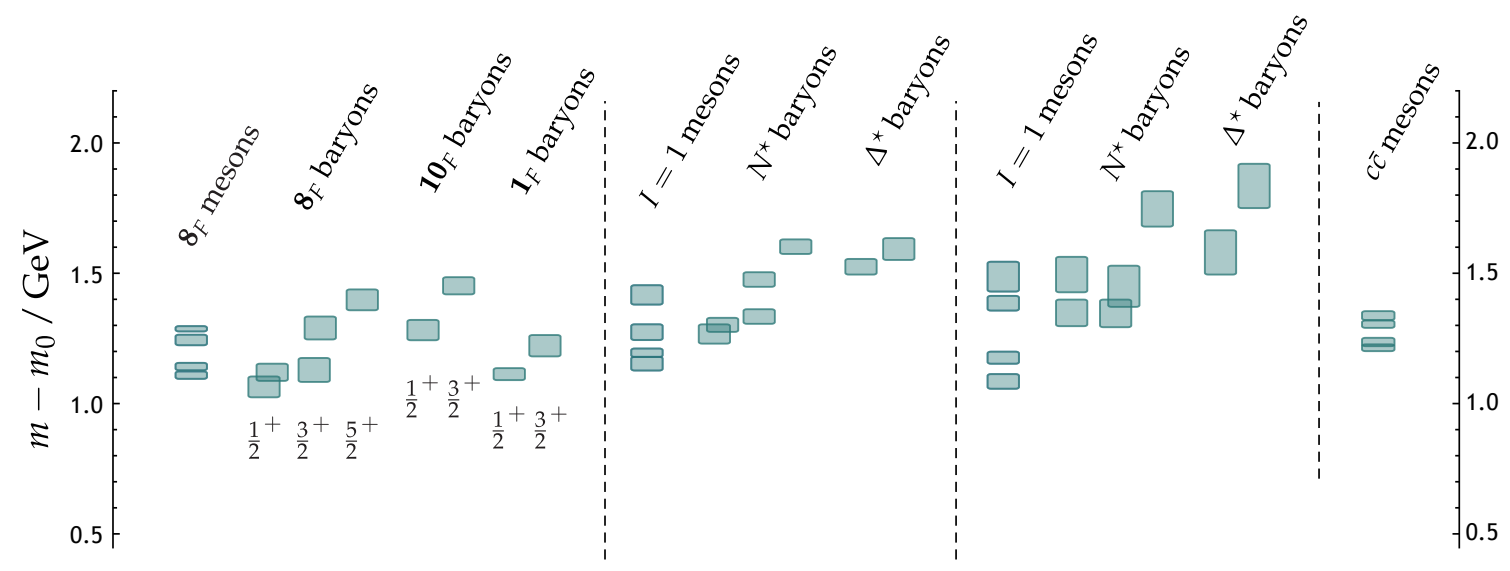

Figure 10: Results from Refs. [64, 65, 66, 13, 67, 68, 69]. Mass scale of states indentified as hybrids versus the pion mass of the ensemble. The results suggest a chromo-magnetic excitation of a color octet with $J^{P C}=1^{+-}$at about an energy scale of $1.3 \mathrm{GeV}$.

constructions. It is also found that the ground-states in these systems also have strong overlap with these chromo-magnetic constructions.

There is a vibrant theory and experimental community investigating the role of glue within nucleon and nuclear systems. What these early spectrum results suggest is that a way to study gluonic content is to probe for these gluonic structures in systems that are not easily accessible experimentally, namely, in excited states, in particular exotic states. In addition, as ground states have a common overlap with this chromo-magnetic induced scale, there could be common features found in Bjorken- $x$ dependent gluonic observables.

The Jefferson Lab $12 \mathrm{GeV}$ program and the Brookhaven RHIC-spin programs are actively investigating the hadronic structure of matter. Lattice QCD calculations can lead a path towards unravelling hadronic structure. Combining the ideas in spectroscopy and hadronic structure studies can guide experimental searches, including those at a possible Electron Ion Collider.

\section{Summary}

Early results from lattice based spectroscopy studies suggests there is a rich spectrum of mesons and baryons, including states with hybrid gluonic content. These results have had direct impact on the JLab experimental program, and have spurred new experimental proposals, including the kaon particle ID upgrade to GlueX, and a new search for hybrid baryons within CLAS12.

In the near term, we can expect to see projects use multiple volumes over a range of pion masses. These investigations will help reveal resonance parameters, including pole locations and couplings to decay channels which can confront as well as guide experimental searches. Knowledge of even the magnitude of branching fractions is useful for experimental analysis.

At higher energies, most resonances decay via coupled channels, including three-body decays. There is active and substantial progress being made in this area. S-matrix formalism is well understood for two-body coupled channels, and while not well understood, we find that development of $\mathrm{S}$-matrix formalism is increasingly important for three-body decays. 
Going forward, there are opportunities as well as challenges. New experimental observations of XYZ states and pentaquark states challenges our conventional understanding of spectroscopy, and we can expect lattice QCD to provide new insights. Understanding of the structure of the states is important. Electromagnetic transition form-factors provide an important tool to investigate structure. With the advent of the formalism for $2+\mathscr{J} \rightarrow 2$ scattering, calculations will allow for the determination of structural informations, e.g., the size, shape, and quark and gluonic content and distribution of resonant states.

Concurrently, there are also exciting progress in the development of near light-cone formalism of hadron and nuclear structure. Directly in Euclidean space, we can compute matrix elements that are approximately equal to light-cone separated matrix elements, and can be systematically improved. A clear signal for the presence of gluonic structures are the existence of exotic mesons. These techniques provide a way to understand the internal structure of states, as well as the role of gluonic structures.

\section{Acknowledgements}

I would like to the thank the organizers of Lattice 2019 in Wuhan for the invitation to give the opening presentation of the meeting. I also want to thank the organizers for a very well run, enjoyable, and stimulating conference. RGE acknowledges support from the U.S. Department of Energy contract DE-AC05-06OR23177, under which Jefferson Science Associates, LLC, manages and operates Jefferson Lab. RGE has received support from the Exascale Computing Project (17-SC20-SC), a collaborative effort of the U.S. Department of Energy Office of Science and the National Nuclear Security Administration. Some of this material is based upon work supported by the U.S. Department of Energy, Office of Science, Office of Advanced Scientific Computing Research and Office of Nuclear Physics, Scientific Discovery through Advanced Computing (SciDAC) program.

\section{References}

[1] M. Luscher and U. Wolff, How to calculate the elastic scattering matrix in two-dimensional quantum field theories by numerical simulation, Nucl. Phys. B339 (1990) 222-252.

[2] M. Luscher, Signatures of unstable particles in finite volume, Nucl. Phys. B364 (1991) 237-254.

[3] K. Rummukainen and S. A. Gottlieb, Resonance scattering phase shifts on a nonrest frame lattice, Nucl.Phys. B450 (1995) 397-436, [hep-lat/ 9503028$].$

[4] C. Kim, C. Sachrajda, and S. R. Sharpe, Finite-volume effects for two-hadron states in moving frames, Nucl.Phys. B727 (2005) 218-243, [hep-lat/ 0507006 ].

[5] N. H. Christ, C. Kim, and T. Yamazaki, Finite volume corrections to the two-particle decay of states with non-zero momentum, Phys.Rev. D72 (2005) 114506, [hep-lat/ 0507009 ].

[6] C. Michael, Adjoint sources in lattice gauge theory, Nucl. Phys. B259 (1985) 58.

[7] B. Blossier, M. Della Morte, G. von Hippel, T. Mendes, and R. Sommer, On the generalized eigenvalue method for energies and matrix elements in lattice field theory, JHEP 04 (2009) 094, [arXiv:0902.1265]. 
[8] J. J. Dudek, R. G. Edwards, M. J. Peardon, D. G. Richards, and C. E. Thomas, Highly excited and exotic meson spectrum from dynamical lattice QCD, Phys. Rev. Lett. 103 (2009) 262001, [arXiv:0909.0200].

[9] J. J. Dudek et al., Toward the excited meson spectrum of dynamical QCD, Phys. Rev. D82 (2010) 034508, [arXiv:1004.4930].

[10] J. J. Dudek, R. G. Edwards, B. Joo, M. J. Peardon, D. G. Richards, et al., Isoscalar meson spectroscopy from lattice QCD, Phys.Rev. D83 (2011) 111502, [arXiv: 1102 . 4299].

[11] C. E. Thomas, R. G. Edwards, and J. J. Dudek, Helicity operators for mesons in flight on the lattice, Phys. Rev. D85 (2012) 014507, [arXiv: 1107.1930$].$

[12] L. Liu et al., Excited and exotic charmonium spectroscopy from lattice QCD, JHEP 1207 (2012) 126, [arXiv:1204.5425].

[13] R. G. Edwards, J. J. Dudek, D. G. Richards, and S. J. Wallace, Excited state baryon spectroscopy from lattice QCD, Phys.Rev. D84 (2011) 074508, [arXiv:1104.5152].

[14] J. J. Dudek and R. G. Edwards, Hybrid baryons in QCD, Phys.Rev. D85 (2012) 054016 , [arXiv:1201.2349].

[15] CP-PACS Collaboration, S. Aoki et al., Lattice QCD Calculation of the rho Meson Decay Width, Phys. Rev. D76 (2007) 094506, [arXiv: 0708 . 370 5].

[16] X. Feng, K. Jansen, and D. B. Renner, Resonance Parameters of the rho-Meson from Lattice QCD, Phys. Rev. D83 (2011) 094505, [arXiv: 1011.5288 ].

[17] C. B. Lang, D. Mohler, S. Prelovsek, and M. Vidmar, Coupled channel analysis of the rho meson decay in lattice QCD, Phys. Rev. D84 (2011), no. 5 054503, [arXiv: 1105.5636 ]. [Erratum: Phys. Rev.D89,no.5,059903(2014)].

[18] C. Pelissier and A. Alexandru, Resonance parameters of the rho-meson from asymmetrical lattices, Phys. Rev. D87 (2013), no. 1 014503, [arXiv:1211.0092].

[19] Z. Fu, Lattice QCD study of the s-wave $\pi \pi$ scattering lengths in the $I=0$ and 2 channels, Phys. Rev. D87 (2013), no. 7 074501, [arXiv:1303.0517].

[20] Hadron Spectrum Collaboration, J. J. Dudek, R. G. Edwards, C. E. Thomas, and D. J. Wilson, Resonances in coupled $\pi K-\eta K$ scattering from quantum chromodynamics, Phys. Rev. Lett. 113 (2014), no. 18 182001, [arXiv:1406.4158].

[21] D. J. Wilson, J. J. Dudek, R. G. Edwards, and C. E. Thomas, Resonances in coupled $\pi K, \eta K$ scattering from lattice QCD, Phys. Rev. D91 (2015), no. 5 054008, [arXiv: 1411.2004 ].

[22] RQCD Collaboration, G. S. Bali, S. Collins, A. Cox, G. Donald, M. GÃúckeler, C. B. Lang, and A. Sch Ãd'fer, $\rho$ and $K^{*}$ resonances on the lattice at nearly physical quark masses and $N_{f}=2$, Phys. Rev. D93 (2016), no. 5 054509, [arXiv: 1512 . 08678 ].

[23] J. Bulava, B. Fahy, B. HÃúrz, K. J. Juge, C. Morningstar, and C. H. Wong, $I=1$ and $I=2 \pi-\pi$ scattering phase shifts from $N_{\mathrm{f}}=2+1$ lattice QCD, Nucl. Phys. B910 (2016) 842-867, [arXiv:1604.05593].

[24] Hadron Spectrum Collaboration, M. Peardon, J. Bulava, J. Foley, C. Morningstar, J. Dudek, R. G. Edwards, B. Joo, H.-W. Lin, D. G. Richards, and K. J. Juge, A Novel quark-field creation operator construction for hadronic physics in lattice QCD, Phys. Rev. D80 (2009) 054506, [arXiv:0905.2160]. 
[25] C. Morningstar, J. Bulava, J. Foley, K. J. Juge, D. Lenkner, M. Peardon, and C. H. Wong, Improved stochastic estimation of quark propagation with Laplacian Heaviside smearing in lattice QCD, Phys. Rev. D83 (2011) 114505, [arXiv: 1104.3870 ].

[26] D. J. Wilson, J. J. Dudek, R. G. Edwards, and C. E. Thomas, Resonances in coupled $\pi K, \eta K$ scattering from lattice QCD, Phys. Rev. D91 (2015), no. 5 054008, [arXiv: 1411.2004 ].

[27] B. HÃûrz and A. Hanlon, Two-and three-pion finite-volume spectra at maximal isospin from lattice QCD, Phys. Rev. Lett. 123 (2019), no. 14 142002, [arXiv: 1905.04277 ].

[28] S. He, X. Feng, and C. Liu, Two particle states and the s-matrix elements in multi-channel scattering, Journal of High Energy Physics 2005 (Jul, 2005) 011âĂŞ011.

[29] M. T. Hansen and S. R. Sharpe, Multiple-channel generalization of Lellouch-Luscher formula, Phys. Rev. D86 (2012) 016007, [arXiv: 1204.0826 ].

[30] R. A. Briceno and Z. Davoudi, Moving multichannel systems in a finite volume with application to proton-proton fusion, Phys. Rev. D88 (2013), no. 9 094507, [arXiv: 1204.1110 ].

[31] R. A. Briceno and Z. Davoudi, Three-particle scattering amplitudes from a finite volume formalism, Phys. Rev. D87 (2013), no. 9 094507, [arXiv:1212.3398].

[32] P. Guo, J. Dudek, R. Edwards, and A. P. Szczepaniak, Coupled-channel scattering on a torus, Phys. Rev. D88 (2013), no. 1 014501, [arXiv:1211.0929].

[33] R. A. Briceno, J. J. Dudek, and R. D. Young, Scattering processes and resonances from lattice QCD, Rev. Mod. Phys. 90 (2018), no. 2 025001, [arXiv: 1706.06223 ].

[34] D. J. Wilson, R. A. Briceno, J. J. Dudek, R. G. Edwards, and C. E. Thomas, Coupled $\pi \pi, K \bar{K}$ scattering in P-wave and the $\rho$ resonance from lattice $Q C D$, Phys. Rev. D92 (2015), no. 9094502 , [arXiv:1507.02599].

[35] R. A. Briceño, M. T. Hansen, and S. R. Sharpe, Relating the finite-volume spectrum and the two-and-three-particle S-matrix for relativistic systems of identical scalar particles, arXiv:1701.07465.

[36] Hadron Spectrum Collaboration, J. J. Dudek, R. G. Edwards, C. E. Thomas, and D. J. Wilson, Resonances in coupled $\pi K-\eta K$ scattering from quantum chromodynamics, Phys. Rev. Lett. 113 (2014), no. 18 182001, [arXiv:1406.4158].

[37] Hadron Spectrum Collaboration, J. J. Dudek, R. G. Edwards, and D. J. Wilson, An a resonance in strongly coupled $\pi \eta, K \bar{K}$ scattering from lattice QCD, Phys. Rev. D93 (2016), no. 9 094506, [arXiv:1602.05122].

[38] R. A. Briceno, J. J. Dudek, R. G. Edwards, and D. J. Wilson, Isoscalar $\pi \pi$ scattering and the $\sigma$ meson resonance from QCD, Phys. Rev. Lett. 118 (2017), no. 2 022002, [arXiv: 1607.05900 ].

[39] R. A. Briceno, J. J. Dudek, R. G. Edwards, and D. J. Wilson, Isoscalar $\pi \pi, K \bar{K}, \eta \eta$ scattering and the $\sigma, f_{0}, f_{2}$ mesons from QCD, Phys. Rev. D97 (2018), no. 5 054513, [arXiv: 1708.06667 ].

[40] D. J. Wilson, R. A. Briceno, J. J. Dudek, R. G. Edwards, and C. E. Thomas, Coupled $\pi \pi, K \bar{K}$ scattering in P-wave and the $\rho$ resonance from lattice $Q C D$, Phys. Rev. D92 (2015), no. 9094502 , [arXiv:1507.02599].

[41] D. J. Wilson, R. A. Briceno, J. J. Dudek, R. G. Edwards, and C. E. Thomas, The quark-mass dependence of elastic $\pi K$ scattering from QCD, Phys. Rev. Lett. 123 (2019), no. 4042002 , [arXiv:1904.03188]. 
[42] R. Brett, J. Bulava, J. Fallica, A. Hanlon, B. HÃû́rz, and C. Morningstar, Determination of $s$ - and p-wave $I=1 / 2 K \pi$ scattering amplitudes in $N_{\mathrm{f}}=2+1$ lattice QCD, Nucl. Phys. B932 (2018) 29-51, [arXiv:1802.03100].

[43] S. Piemonte, S. Collins, D. Mohler, M. Padmanath, and S. Prelovsek, Charmonium resonances with $J^{P C}=1^{--}$and $3^{--}$from $\bar{D} D$ scattering on the lattice, Phys. Rev. D100 (2019), no. 7074505 , [arXiv:1905.03506].

[44] Hadron Spectrum Collaboration, G. K. C. Cheung, C. E. Thomas, J. J. Dudek, and R. G. Edwards, Tetraquark operators in lattice QCD and exotic flavour states in the charm sector, JHEP 11 (2017) 033, [arXiv:1709.01417].

[45] X. Feng, S. Aoki, S. Hashimoto, and T. Kaneko, Timelike pion form factor in lattice QCD, Phys. Rev. D91 (2015), no. 5 054504, [arXiv: 1412.6319 ].

[46] C. Andersen, J. Bulava, B. HÃuurz, and C. Morningstar, The I=1 pion-pion scattering amplitude and timelike pion form factor from $N_{\mathrm{f}}=2+1$ lattice QCD, Nucl. Phys. B939 (2019) 145-173, [arXiv:1808.05007].

[47] R. A. Briceno, J. J. Dudek, R. G. Edwards, C. J. Shultz, C. E. Thomas, and D. J. Wilson, The resonant $\pi^{+} \gamma \rightarrow \pi^{+} \pi^{0}$ amplitude from Quantum Chromodynamics, Phys. Rev. Lett. 115 (2015) 242001, [arXiv:1507.06622].

[48] R. A. Brice Ãśo, J. J. Dudek, R. G. Edwards, C. J. Shultz, C. E. Thomas, and D. J. Wilson, The $\pi \pi \rightarrow \pi \gamma^{\star}$ amplitude and the resonant $\rho \rightarrow \pi \gamma^{\star}$ transition from lattice QCD, Phys. Rev. D93 (2016), no. 11 114508, [arXiv:1604.03530].

[49] R. A. Briceño and M. T. Hansen, Multichannel $0 \rightarrow 2$ and $1 \rightarrow 2$ transition amplitudes for arbitrary spin particles in a finite volume, Phys. Rev. D92 (2015), no. 7 074509, [arXiv: 1502.04314 ].

[50] R. A. Briceño, M. T. Hansen, and A. Walker-Loud, Multichannel $1 \rightarrow 2$ transition amplitudes in a finite volume, Phys. Rev. D91 (2015), no. 3 034501, [arXiv:1406.5965].

[51] LHCb Collaboration, R. Aaij et al., Observation of J/ $\psi$ p Resonances Consistent with Pentaquark States in $\Lambda_{b}^{0} \rightarrow J / \psi K^{-}$p Decays, Phys. Rev. Lett. 115 (2015) 072001, [arXiv: 1507.03414 ].

[52] LHCb Collaboration, R. Aaij et al., Model-independent evidence for J/ $\psi$ p contributions to $\Lambda_{b}^{0} \rightarrow J / \psi p K^{-}$decays, Phys. Rev. Lett. 117 (2016), no. 8 082002, [arXiv: 1604.05708 ].

[53] LHCb Collaboration, R. Aaij et al., Observation of a narrow pentaquark state, $P_{c}(4312)^{+}$, and of two-peak structure of the $P_{c}(4450)^{+}$, Phys. Rev. Lett. 122 (2019), no. 22 222001, [arXiv:1904.03947].

[54] GlueX Collaboration, A. Ali et al., First Measurement of Near-Threshold J/ÏL Exclusive Photoproduction off the Proton, Phys. Rev. Lett. 123 (2019), no. 7 072001, [arXiv: 1905.10811 ].

[55] A. Baroni, R. A. BriceÃśo, M. T. Hansen, and F. G. Ortega-Gama, Form factors of two-hadron states from a covariant finite-volume formalism, Phys. Rev. D100 (2019), no. 3 034511, [arXiv:1812.10504].

[56] R. A. BriceÃśo, J. V. Guerrero, M. T. Hansen, and C. J. Monahan, Finite-volume effects due to spatially nonlocal operators, Phys. Rev. D98 (2018), no. 1 014511, [arXiv: 1805.01034 ].

[57] R. A. Brice Ãśo, M. T. Hansen, and S. R. Sharpe, Three-particle systems with resonant subprocesses in a finite volume, Phys. Rev. D99 (2019), no. 1 014516, [arXiv: 1810.01429 ]. 
[58] R. A. Briceño, M. T. Hansen, S. R. Sharpe, and A. P. Szczepaniak, Unitarity of the infinite-volume three-particle scattering amplitude arising from a finite-volume formalism, Phys. Rev. D100 (2019), no. 5 054508, [arXiv:1905.11188].

[59] A. W. Jackura, S. M. Dawid, C. FernÃąndez-RamÃ S. R. Sharpe, and A. P. Szczepaniak, Equivalence of three-particle scattering formalisms, Phys. Rev. D100 (2019), no. 3 034508, [arXiv: 1905.12007 ].

[60] U.-G. MeiÃßner, G. RÃ̄os, and A. Rusetsky, Spectrum of three-body bound states in a finite volume, Phys. Rev. Lett. 114 (2015), no. 9 091602, [arXiv:1412.4969]. [Erratum: Phys. Rev. Lett.117,no.6,069902(2016)].

[61] Y. Meng, C. Liu, U.-G. MeiÃßner, and A. Rusetsky, Three-particle bound states in a finite volume: unequal masses and higher partial waves, Phys. Rev. D98 (2018), no. 1014508 , [arXiv:1712.08464].

[62] J.-Y. Pang, J.-J. Wu, H. W. Hammer, U.-G. MeiÃ§ner, and A. Rusetsky, Energy shift of the three-particle system in a finite volume, Phys. Rev. D99 (2019), no. 7 074513, [arXiv:1902.01111].

[63] A. J. Woss, C. E. Thomas, J. J. Dudek, R. G. Edwards, and D. J. Wilson, $b_{1}$ resonance in coupled $\pi \omega$, $\pi \phi$ scattering from lattice QCD, Phys. Rev. D100 (2019), no. 5 054506, [arXiv: 1904.04136 ].

[64] J. J. Dudek, R. G. Edwards, M. J. Peardon, D. G. Richards, and C. E. Thomas, Highly excited and exotic meson spectrum from dynamical lattice QCD, Phys. Rev. Lett. 103 (2009) 262001, [arXiv:0909.0200].

[65] J. J. Dudek, The lightest hybrid meson supermultiplet in QCD, Phys. Rev. D84 (2011) 074023, [arXiv:1106.5515].

[66] J. J. Dudek, R. G. Edwards, M. J. Peardon, D. G. Richards, and C. E. Thomas, Toward the excited meson spectrum of dynamical QCD, Phys. Rev. D82 (2010) 034508, [arXiv: 1004 . 4930].

[67] J. J. Dudek and R. G. Edwards, Hybrid Baryons in QCD, Phys. Rev. D85 (2012) 054016, [arXiv:1201.2349].

[68] Hadron Spectrum Collaboration, L. Liu, G. Moir, M. Peardon, S. M. Ryan, C. E. Thomas, P. Vilaseca, J. J. Dudek, R. G. Edwards, B. Joo, and D. G. Richards, Excited and exotic charmonium spectroscopy from lattice QCD, JHEP 07 (2012) 126, [arXiv: 1204.5425 ].

[69] Hadron Spectrum Collaboration, J. J. Dudek, R. G. Edwards, P. Guo, and C. E. Thomas, Toward the excited isoscalar meson spectrum from lattice QCD, Phys.Rev. D88 (2013), no. 9 094505, [arXiv:1309.2608]. 